\title{
A measurement of the cosmological mass density from clustering in the 2dF Galaxy Redshift Survey
} \author{
Matthew Colless ${ }^{4}$, Chris Collins ${ }^{5}$, Warrick Couch ${ }^{6}$, Gavin Dalton ${ }^{7}$, Kathryn Deeley ${ }^{6}$, Roberto De Propris ${ }^{6}$, Simon P. Driver $^{8}$, \\ Steve Maddox ${ }^{13}$, Will J. Percival ${ }^{1}$, Bruce A. Peterson ${ }^{4}$, lan Price ${ }^{4}$, Will Sutherland ${ }^{1,7}$ \& Keith Taylor ${ }^{3,10}$

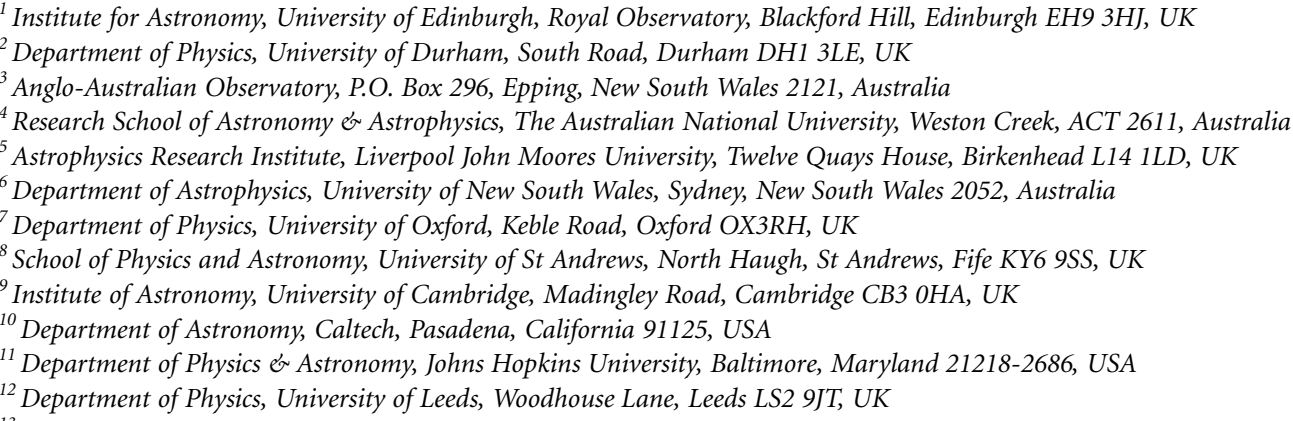

John A. Peacock', Shaun Cole ${ }^{2}$, Peder Norberg' ${ }^{2}$, Carlton M. Baugh' ${ }^{2}$, Joss Bland-Hawthorn ${ }^{3}$, Terry Bridges ${ }^{3}$, Russell D. Cannon ${ }^{3}$, George Efstathiou ${ }^{9}$, Richard S. Ellis ${ }^{9,10}$, Carlos S. Frenk ${ }^{2}$, Karl Glazebrook ${ }^{11}$, Carole Jackson ${ }^{4}$, Ofer Lahav ${ }^{9}$, lan Lewis ${ }^{3}$, Stuart Lumsden ${ }^{12}$,

The large-scale structure in the distribution of galaxies is thought to arise from the gravitational instability of small fluctuations in the initial density field of the Universe. A key test of this hypothesis is that forming superclusters of galaxies should generate a systematic infall of other galaxies. This would be evident in the pattern of recessional velocities, causing an anisotropy in the inferred spatial clustering of galaxies. Here we report a precise measurement of this clustering, using the redshifts of more than 141,000 galaxies from the two-degree-field ( $2 \mathrm{dF}$ ) galaxy redshift survey. We determine the parameter $\beta=\Omega^{0.6} / b=0.43 \pm 0.07$, where $\Omega$ is the total mass-density parameter of the Universe and $b$ is a measure of the 'bias' of the luminous galaxies in the survey. (Bias is the difference between the clustering of visible galaxies and of the total mass, most of which is dark.) Combined with the anisotropy of the cosmic microwave background, our results favour a low-density Universe with $\Omega \approx 0.3$.

Hubble showed in 1934 that the pattern of galaxies on the sky is non-random ${ }^{1}$, and successive years have seen ever more ambitious attempts to map the distribution of visible matter on cosmological scales. In order to obtain a three-dimensional picture, redshift surveys use Hubble's law, $v=H_{0} r$, to infer approximate radial distances to a set of galaxies. The first major surveys of this sort took place in the early 1980s (refs $2-5$ ), and were limited to a few thousand redshifts, owing to the limited speed of single-object spectroscopy. In the 1990s, redshift surveys were extended to much larger volumes by a 'sparse sampling' strategy ${ }^{6}$. These studies ${ }^{7,8}$ established that the universe was close to uniform on scales above about $100 h^{-1} \mathrm{Mpc}$ (where $h \equiv H_{0} / 100 \mathrm{~km} \mathrm{~s}^{-1} \mathrm{Mpc}^{-1}$ ), but with a complex nonlinear supercluster network of walls, filaments and voids on smaller scales.

The origin of this large-scale structure is one of the key issues in cosmology. A plausible assumption is that structure grows by gravitational collapse of density fluctuations that are small at early times-but it is essential to test this idea. One important signature of gravitational instability is that collapsing structures should generate 'peculiar' velocities, $\delta \mathbf{v}$, which distort the uniform Hubble expansion. We measure a redshift, $z$, which combines Hubble's law with the radial component of these peculiar velocities: $c z \simeq H_{0} r+\delta \mathbf{v} \cdot \hat{\mathbf{r}}$. The apparent density field seen in a redshift survey in thus not a true three-dimensional picture, but this can be turned to our advantage. The redshift-space distortions have a characteristic form, whose detection can both verify the general idea that structure forms by gravitational instability, and also measure the density of the universe. Here we present measurements of this effect, based on a new large redshift survey.

\section{The 2dF Galaxy Redshift Survey}

New-generation large redshift surveys are made feasible by multiplexed fibre-optic spectroscopy, and the most advanced facility of this sort is the two-degree field, mounted at the prime focus of the Anglo-Australian Telescope ${ }^{9}$, which allows 400 spectra to be measured simultaneously. (For details of the $2 \mathrm{df}$ instrument, see http://www.aao.gov.au/2df/.) The 2dF Galaxy Redshift Survey $(2 \mathrm{dFGRS})^{10}$ was designed to use this instrument to measure the redshifts of 250,000 galaxies, to a blue magnitude limit of $b_{\mathrm{I}}=19.45$ (corrected for extinction by dust in the Milky Way). (For details of the current status of the 2dFGRS, see http://www.mso.anu.edu.au/ 2dFGRS/.) The galaxies were selected from an updated version of the APM (automatic plate-measuring machine) catalogue ${ }^{11}$, which is based on scans of photographic plates taken with the UK Schmidt telescope. Survey observations began in 1998, and should finish at the end of 2001. Redshifts have currently been obtained for 141,402 galaxies. The sky coverage of the $2 \mathrm{dFGRS}$ consists of strips in the northern and southern galactic poles $\left(75^{\circ} \times 7.5^{\circ}\right.$ in the NGP; $75^{\circ} \times 15^{\circ}$ in the SGP), plus a number of outlying random fields. Coverage is now sufficiently extensive that near-complete thin slices through the galaxy distribution may be constructed, as shown in Fig. 1. This image illustrates well the median depth of the survey: approximately $z=0.11$. Beyond this point, the survey is sensitive only to the more luminous galaxies, and the comoving density falls 
rapidly. Nevertheless, the survey volume (including other regions not shown) is more than adequate for an accurate determination of the statistical properties of galaxy clustering.

\section{Galaxy correlations in redshift space}

The simplest statistical indicator of peculiar velocities in cosmological structure is the two-point correlation function, $\xi(\sigma, \pi)$. This measures the excess probability over random probability of finding a pair of galaxies with a transverse separation $\sigma$ and a line-of-sight separation $\pi$. In an isotropic universe, this function should be independent of direction, but this is not true in redshift space. Transverse separations are true measures of distance, but apparent radial separations are distorted by peculiar velocities. This redshiftspace anisotropy should cause two characteristic effects, operating respectively on small and large scales. On small scales, random orbital velocities within galaxy groups cause an apparent radial smearing, known as 'fingers of God'. Of greater interest is the largescale effect; if cosmological structure forms by gravitational collapse, there should exist coherent infall velocities, and the effect of these is to cause an apparent flattening of structures along the line of sight. The general existence of redshift-space distortions was recognized in the first redshift surveys ${ }^{2-4}$, but the first comprehensive analysis of the phenomenon was performed by Kaiser ${ }^{12}$, who showed that they could be used to measure the quantity

$$
\beta \equiv \Omega^{0.6} / b
$$

where $\Omega$ is the cosmological mass density parameter and $b$ is the bias parameter that relates the relative density fluctuations of the galaxies and of the total mass:

$$
\left.\frac{\delta \rho}{\rho}\right|_{\text {galaxies }}=\left.b \frac{\delta \rho}{\rho}\right|_{\text {mass }}
$$

The presence of bias is an inevitable consequence of the nonlinear nature of galaxy formation, and the relation between mass and galaxy tracers is complex ${ }^{13-15}$. However, there are good theoretical reasons to expect that $b$ can indeed be treated as a constant on large scales, where the density fluctuations are linear ${ }^{16,17}$. Redshift-space distortions have thus been seen as an important method for weighing the universe ${ }^{18,19}$. To date, a number of papers have made significant detections of the Kaiser effect ${ }^{13,20,21}$, but the 2dFGRS is the first survey that is large enough for the effect to be studied in detail.

In order to estimate $\xi(\sigma, \pi)$, we follow standard methods ${ }^{22,23}$ that compare the observed count of galaxy pairs with the count estimated using a random distribution that obeys the same selection effects in redshift and sky position. These selection effects are well defined, but complex: the survey is tessellated into a pattern of 'sectors' defined by the overlap of the $2^{\circ}$ diameter survey tiles, whose positions are chosen adaptively with the aim of being able to place a fibre on more than $95 \%$ of the galaxies in the input catalogue. At the present intermediate stage of the survey, many tiles remain to be observed, and some regions of the survey presently contain redshifts for fewer than $50 \%$ of the galaxies. Furthermore, the spectroscopic success rate (redshifts per allocated fibre) is more than $95 \%$ in good conditions, but can fall to about $80 \%$ in marginal weather. We have implemented a number of independent algorithms for estimating the resulting survey selection effects, and are confident that we can measure the galaxy correlations robustly out to a separation of $25 h^{-1} \mathrm{Mpc}$. For example, the redshift distribution in sectors with low spectroscopic completeness is biased to low redshifts, but it makes no significant difference whether or not we correct for this, or indeed whether the low-completeness regions are simply excised. In addition to allowing for survey completeness, it is necessary to give higher weight to regions with a low sampling density, to achieve the optimum balance between cosmic variance and shot noise ${ }^{6}$. In practice, we have chosen to truncate the analysis at a maximum redshift of $z=0.25$. Within this volume, the exact optimum weight per galaxy varies very nearly as the reciprocal of the number density, so that all volume elements receive approximately equal weight. The redshift-space correlation function for the 2dFGRS computed in this way is shown in Fig. 2. The correlation-function results display very clearly the two signatures of redshift-space distortions discussed above. The 'fingers of God' from small-scale random velocities are very clear, as indeed has been the case from the first redshift surveys ${ }^{3}$. However, this is the first time that the detailed

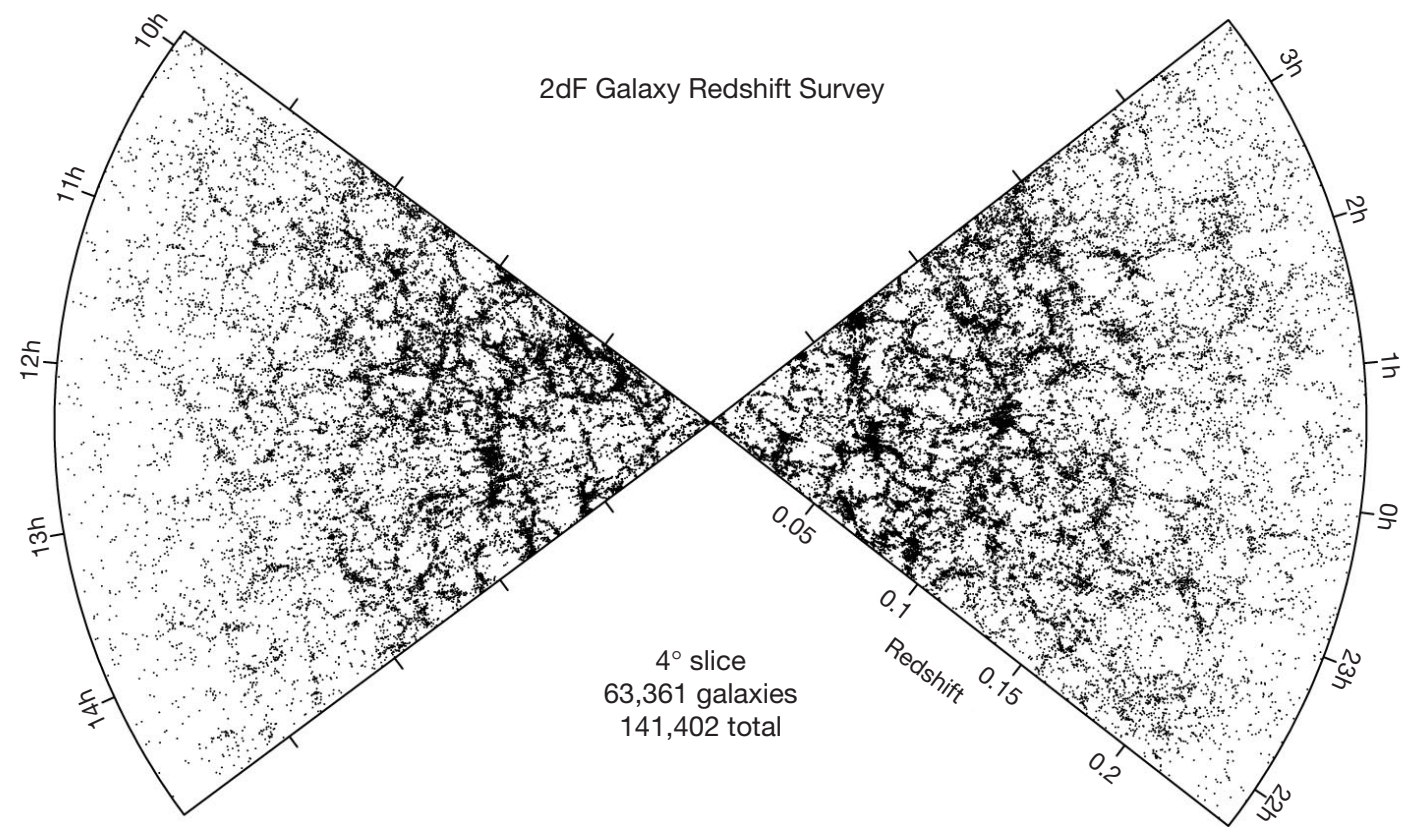

Figure 1 The distribution of galaxies in part of the two-degree-field galaxy redshift survey (2dFGRS), drawn from a total of 141,402 galaxies. The slices are $4^{\circ}$ thick, centred at declination $-2.5^{\circ}$ in the Northern Galactic Pole (left) and $-27.5^{\circ}$ in the Southern Galactic Pole (right). Not all 2 df fields within the slice have been observed at this stage; hence there are weak variations of the density of sampling as a function of right ascension. To minimize such features, the slice thickness increases to $7.5^{\circ}$ between right ascension $13.1 \mathrm{~h}$ and $13.4 \mathrm{~h}$. This image reveals a wealth of detail, including linear supercluster features, often nearly perpendicular to the line of sight. The interesting question to settle statistically is whether such transverse features have been enhanced by infall velocities. 
signature of large-scale flattening from coherent infall has been seen with a high signal-to-noise ratio.

\section{Quantifying redshift-space distortions}

The large-scale flattening of the correlation function may be quantified by measuring the quadrupole moment of $\xi(\sigma, \pi)$ as a function of radius $r$. A negative quadrupole moment implies flattening, whereas the finger-of-God distortion tends to yield a positive quadrupole moment. Figure 3 shows that the quadrupoleto-monopole ratio is positive on small scales, but that if falls with separation, becoming progressively more negative out to the largest separations at which it can be reliably measured. This arises partly because the underlying power spectrum is not a simple power-law function of scale, so that the peculiar velocities have a different effect at different radii. By integrating over the correlation function, it is possible to construct quantities in which this effect is eliminated. We shall not do this here, firstly because it seems desirable to keep the initial analysis as direct as possible. More importantly, finger-ofGod smearing is a significant correction that will also cause the flattening to depend on radius. We therefore have to fit the data with a two-parameter model, described in the caption to Fig. 2. The parameters are $\beta$ and a measure of the size of the random dispersion

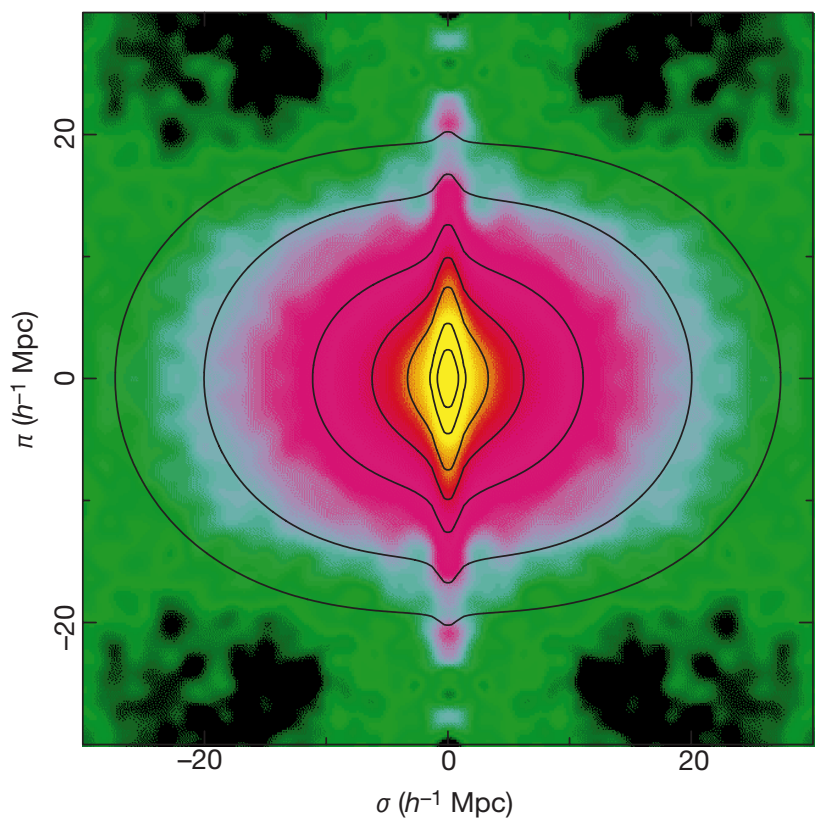

Figure 2 The redshift-space correlation function for the 2dFGRS, $\xi(\sigma, \pi)$, plotted as a function of transverse $(\sigma)$ and radial $(\pi)$ pair separation. The function was estimated by counting pairs in boxes of side $0.2 \mathrm{~h}^{-1} \mathrm{Mpc}$, and then smoothing with a gaussian of r.m.s. width $0.5 h^{-1} \mathrm{Mpc}$. To illustrate deviations from circular symmetry, the data from the first quadrant are repeated with reflection in both axes. This plot clearly displays redshift distortions, with finger-of-God elongations at small scales and the coherent Kaiser flattening at large radii. The overplotted contours show model predictions with flattening parameter $\beta \equiv \Omega^{0.6} / b=0.4$ and a pairwise dispersion of $\sigma_{\mathrm{p}}=400 \mathrm{~km} \mathrm{~s}^{-1}$. Contours are plotted at $\xi=10,5,2,1,0.5,0.2$ and 0.1 .

The model predictions assume that the redshift-space power spectrum $\left(P_{\mathrm{s}}\right)$ may be expressed as a product of the linear Kaiser distortion and a radial convolution ${ }^{37}$ :

$P_{\mathrm{s}}(\mathbf{k})=P_{r}(k)\left(1+\beta \mu^{2}\right)^{2}\left(1+k^{2} \sigma_{\mathrm{p}}^{2} \mu^{2} / 2 H_{0}^{2}\right)^{-1}$, where $\mu=\hat{\mathbf{k}} \cdot \hat{\mathbf{r}}$, and $\sigma_{\mathrm{p}}$ is the r.m.s. pairwise dispersion of the random component of the galaxy velocity field. This model gives a very accurate fit to exact nonlinear simulations ${ }^{33}$. For the real-space power spectrum, $P_{\mathrm{r}}(k)$, we take the estimate obtained by deprojecting the angular clustering in the APM survey ${ }^{11,39}$. This agrees very well with estimates that can be made directly from the 2dFGRS, as will be discussed elsewhere. We use this model only to estimate the scale dependence of the quadrupole-to-monopole ratio (although Fig. 2 shows that it does match the full $\xi(\sigma, \pi)$ data very well). in the relative velocities of galaxies, $\sigma_{\mathrm{p}}$. In practice, $\sigma_{\mathrm{p}}$ plays the role of an empirical fitting parameter to describe the scale on which the distortions approach the linear-theory predictions. It therefore also incorporates other possible effects, such as a scale dependence of bias.

The results for the quadrupole-to-monopole ratio are shown in Fig. 3, which shows the average of the estimates for the NGP and SGP slices. The difference between the NGP and SGP allows an estimate of the errors to be made: these slices are independent samples for the present analysis of clustering on relatively small scales. For model fitting, it is necessary to know the correlation between the values at different $r$. A simple way of addressing this is to determine the effective number of degrees of freedom from the value of $\chi^{2}$ for the best-fitting model. A more sophisticated approach is to generate realizations of $\xi(\sigma, \pi)$, and construct the required covariance matrix directly. One way of achieving this is to analyse large numbers of mock surveys drawn from numerical simulations ${ }^{24}$. A more convenient method is to generate direct realizations of the redshift-space power spectrum, using gaussian fluctuations on large scales, but allowing for enhanced variance in power on nonlinear scales ${ }^{25-27}$. In practice, the likelihood contours resulting from this approach agree well with those from the simple approach, and we are confident that the resulting errors on $\beta$ are realistic. These contours are shown in Fig. 4, and show that there is a degree of correlation between the preferred values of $\beta$ and $\sigma_{\mathrm{p}}$, as expected. For our purposes, $\sigma_{\mathrm{p}}$ is an uninteresting parameter, so we marginalize over it to obtain the following estimate of $\beta$ and its root mean square (r.m.s.) uncertainty:

$$
\beta=0.43 \pm 0.07
$$

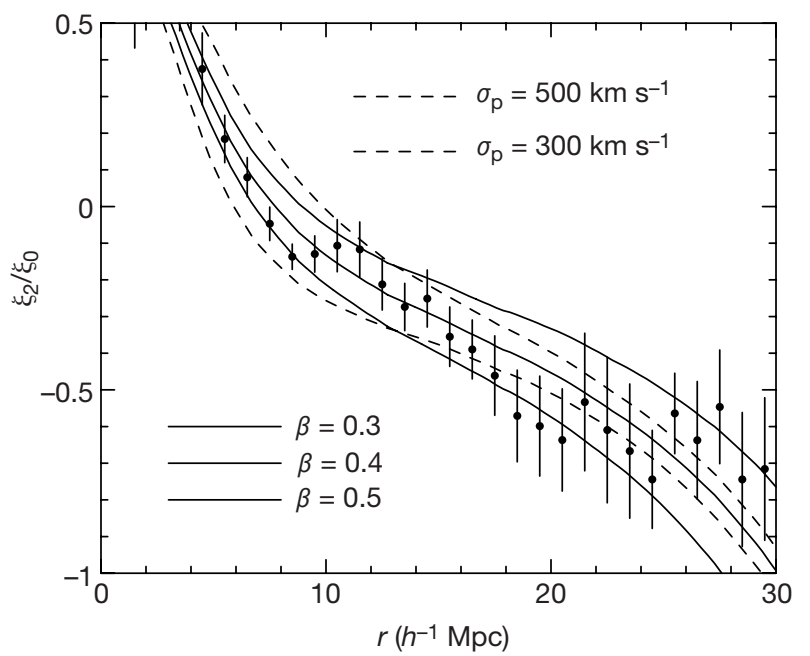

Figure 3 The flattening of the redshift-space correlation function is quantified by the quadrupole-to-monopole ratio, $\xi_{2} / \xi_{0}$. This quantity is positive where fingers-of-God distortion dominates, and is negative where coherent infall dominates. The solid lines show model predictions for $\beta=0.3,0.4$ and 0.5 , with a pairwise velocity dispersion of $\sigma_{\mathrm{p}}=400 \mathrm{~km} \mathrm{~s}^{-1}$ (solid lines), plus $\beta=0.4$ with $\sigma_{\mathrm{p}}=300$ and $500 \mathrm{~km} \mathrm{~s}^{-1}$ (dashed lines). The $\xi_{2} / \xi_{0}$ ratio becomes more negative as $\beta$ increases and as $\sigma_{p}$ decreases. At large radii, the effects of fingers-of-God become relatively small, and values of $\beta \simeq 0.4$ are clearly appropriate.

The multipole moments of the correlation function are defined as $\xi_{\ell}(r) \equiv(2 \ell+1) /$ $2 \int_{-1}^{1} \xi(\sigma=r \sin \theta, \pi=r \cos \theta) P_{\ell}(\cos \theta) d \cos \theta$. In linear theory, the quadrupole-tomonopole ratio is given ${ }^{40}$ by $\xi_{2} / \xi_{0}=f(n)\left(4 \beta / 3+4 \beta^{2} / 7\right) /\left(1+2 \beta / 3+\beta^{2} / 5\right)$. Here $f(n)=(3+n) / n$, where $n$ is the power-spectrum index of the density fluctuations: $\xi \propto r^{-(3+n)}$. In practice, nonlinear effects mean that this ratio is a function of scale. We model this by using the real-space correlation function estimated from the APM survey ${ }^{11,39}$, plus the model for nonlinear finger-of-God smearing given in the caption to Fig. 2. 
This result is the first precise determination of $\beta$ from redshiftspace distortions. The best previous studies ${ }^{13,20,21}$ have only achieved a detection of the effect at the $3 \sigma$ level. Before discussing the implications of our result, we should therefore consider some possible small systematic corrections that have been unimportant in earlier work. First, the Kaiser analysis applies only in the smallangle approximation, and in principle corrections might be needed for wide-angle surveys such as ours ${ }^{28}$. However, with our weighting scheme, the mean angular separation of pairs with spatial separations less than $30 \mathrm{~h}^{-1} \mathrm{Mpc}$ is only $2.5^{\circ}$, so this is not a concern. There is potentially a significant correction for luminosity effects. The optimal weighting means that our mean luminosity is high: it is approximately $M_{b_{\mathrm{J}}}=-20.3$, or 1.9 times the characteristic luminosity, $L^{\star}$, of the overall galaxy population ${ }^{29}$. Several studies ${ }^{30}$ have suggested that the strength of galaxy clustering increases with luminosity, with an effective bias that can be fitted by $b / b^{*}=0.7+0.3\left(L / L^{*}\right)$. This effect has been controversial ${ }^{31}$, but the 2dFGRS data set favours a similar luminosity dependence, as will be described elsewhere. We therefore expect that $\beta$ for $L^{\star}$ galaxies will exceed our directly measured figure. Applying a correction using the given formula for $b(L)$, we deduce

$$
\beta\left(L=L^{*}\right)=0.54 \pm 0.09
$$

Finally, the 2 dFGRS has a median redshift of 0.11 . With weighting, the mean redshift in the present analysis is $\bar{z}=0.17$, and our measurement should be interpreted as $\beta$ at that redshift. The extrapolation to $z=0$ is model-dependent, but probably does not introduce a significant change $e^{32}$.

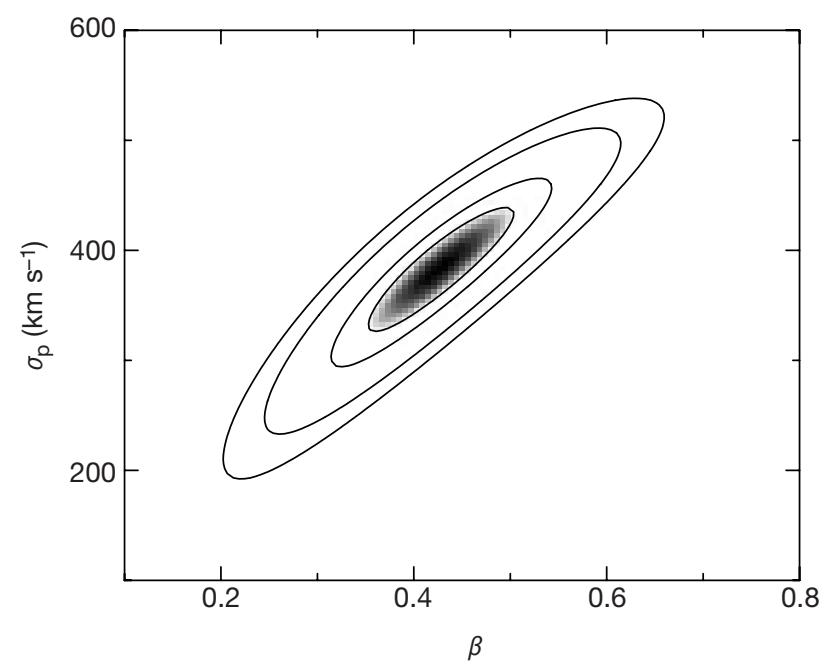

Figure 4 Likelihood contours for $\beta$ and the fingers-of-God smearing parameter $\sigma_{p}$, based on the data in Fig. 3 (considering $8 h^{-1} \mathrm{Mpc}<r<25 h^{-1} \mathrm{Mpc}$ ). These are plotted at the usual positions for one-parameter confidence of $68 \%$ (shaded region), and twoparameter confidence of $68 \%, 95 \%$ and $99 \%$ (that is, $\Delta \chi^{2}=1,2.3,6.0$ and 9.2 ). The maximum-likelihood solution is $\beta=0.43$ and $\sigma_{\mathrm{p}}=385 \mathrm{~km} \mathrm{~s}^{-1}$. The value for the largescale pairwise dispersion is in reasonable agreement with previously suggested values ${ }^{41}$; however, for the present analysis $\sigma_{\mathrm{p}}$ is an uninteresting parameter. If we marginalize over $\sigma_{\mathrm{p}}$ (that is, integrate over $\sigma_{\mathrm{p}}$, treating the likelihood as a probability distribution), the final estimate of $\beta$ and its r.m.s. uncertainty is $\beta=0.43 \pm 0.07$. We believe that this result is robust, in the sense that systematic errors in the modelling are smaller than the random errors. We have tried assuming that the power spectrum for $k<0.1 \mathrm{~h} \mathrm{Mpc}^{-1}$ has the shape of a $\Omega=0.3 \mathrm{CDM}$ model, rather than the APM measurement; this has a very small effect. A more serious issue is whether the pairwise velocity dispersion of galaxies may depend strongly on separation, as is found for mass particles in numerical simulations ${ }^{42}$. Assuming that the pairwise velocity dispersion $\sigma_{p}$ rises to twice its large-scale value below $1 h^{-1} \mathrm{Mpc}$ reduces the best-fit $\beta$ by 0.04 . This correction is small because our analysis excludes the nonlinear data at $r<8 h^{-1} \mathrm{Mpc}$.

\section{Consistency with microwave-background anisotropies}

We have verified in some detail that the pattern of redshift-space distortions associated with the gravitationally driven growth of clustering exists as predicted. Although gravitational instability is well established as the standard model for the formation of largescale structure, it is important to have verified such a characteristic feature of the theory. Extracting the full cosmological implications of our measurement of $\Omega^{0.6} / \mathrm{b}$ requires us to know the bias parameter in order to determine $\Omega$. For example, our measurement implies $\Omega=0.36 \pm 0.10$ if $L^{*}$ galaxies are unbiased, but it is difficult to justify such an assumption. In principle, the details of the clustering pattern in the nonlinear regime allow the $\Omega-b$ degeneracy to be broken, yielding a direct determination of the degree of bias $^{33}$. We expect to pursue this approach using the 2dFGRS. For the present, however, it is interesting to use an independent approach. Observations of anisotropies in the cosmic microwave background $(\mathrm{CMB})$ can in principle measure almost all the cosmological parameters, and current small-scale anisotropy results are starting to tighten the constraints. In a recent analysis ${ }^{34}$, best-fitting values for the densities in collisionless matter, baryons and vacuum (subscripts c, b and v, respectively) have been obtained: $\Omega_{\mathrm{c}}+\Omega_{\mathrm{b}}+\Omega_{\mathrm{v}}=1.11 \pm 0.07, \quad \Omega_{\mathrm{c}} h^{2}=0.14 \pm 0.06$, $\Omega_{\mathrm{b}} h^{2}=0.032 \pm 0.005$, together with a power-spectrum index $n=1.01 \pm 0.09$. Our result for $\beta$ gives an independent test of this picture, as follows.

The only parameter left undetermined by the CMB data is the Hubble constant, $h$. Recent work ${ }^{35,36}$ indicates that this is now determined to an r.m.s. accuracy of $10 \%$, and we adopt a central value of $h=0.70$. This completes the cosmological model, requiring a total matter density parameter $\Omega \equiv \Omega_{\mathrm{c}}+\Omega_{\mathrm{b}}=0.35 \pm 0.14$. It is then possible to use the parameter limits from the CMB to predict a conservative range for the mass power spectrum at $z=0$, which is shown in Fig. 5. In this plot, the mass power spectrum appears to be in good agreement with the clustering observed in the APM survey. For each model allowed by the CMB, we can predict both $b$ (from the ratio of galaxy and mass spectra) and also $\beta$ (because a given CMB model specifies $\Omega$ ). In practice, we determine

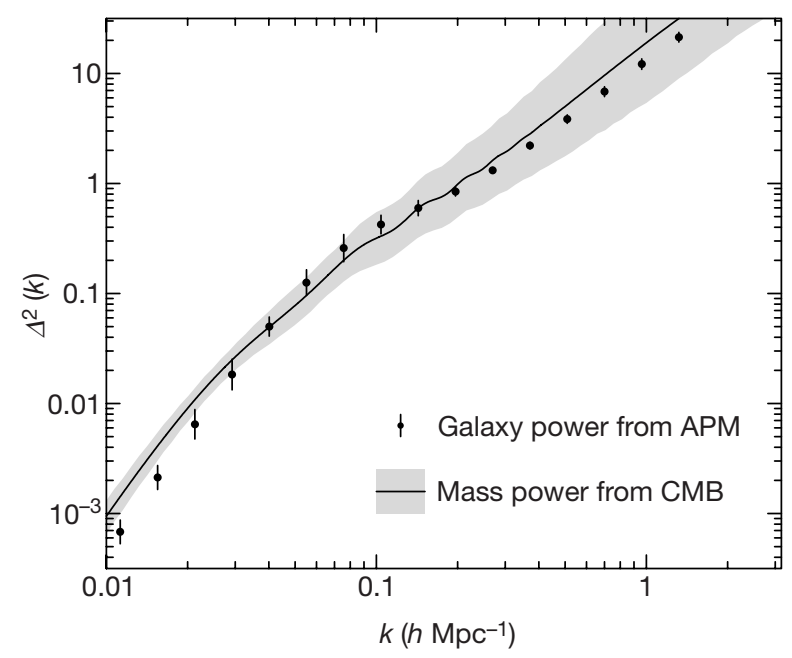

Figure 5 The dimensionless matter power spectrum at zero redshift, $\Delta^{2}(k)$, as predicted from the allowed range of models that fit the microwave-background anisotropy data, plus the assumption that $H_{0}=70 \mathrm{~km} \mathrm{~s}^{-1} \mathrm{Mpc}^{-1} \pm 10 \%$. The solid line shows the best-fit mode $^{34}$ (power-spectrum index $n=1.01$, and density parameters in baryons, CDM, and vacuum of, respectively, $0.065,0.285$ and 0.760 ). The effects of nonlinear evolution have been included ${ }^{43}$. The shaded points are the real-space power spectrum measured for APM galaxies. The clear conclusion is that APM galaxies are consistent with being essentially unbiased tracers of the mass on large scales. As the CMB data also constrain the range of $\Omega$, this allows $\beta$ to be predicted. 
$b$ by determining the mean ratio of power spectra over the range $0.02<k<0.1 \mathrm{~h} \mathrm{Mpc}^{-1}$, where the APM measurement is robust and where scale-dependent bias and nonlinearities should be unimportant. Considering the allowed range of models, we then obtain the prediction $\beta_{\mathrm{CMB}+\mathrm{APM}}=0.57 \pm 0.17$. A flux-limited survey such as the APM will have a mean luminosity close to $L^{*}$, so the appropriate comparison is with the 2dFGRS corrected figure of $\beta=0.54 \pm 0.09$ for $L^{\star}$ galaxies. These numbers are in very close agreement.

This analysis of galaxy clustering in the 2dFGRS thus gives strong support to the simplest picture of cosmological structure formation, in which the primary mechanism is gravitational instability in a sea of collisionless dark matter. We have shown that the fluctuations seen in the CMB (which measure structure at a redshift $z \simeq 1100$ ) can be extrapolated to the present to predict the peculiar velocities that distort redshift-space clustering. The agreement between this extrapolation and direct observations from the 2dFGRS is a highly non-trivial confirmation of the basic model. The precision of data in both areas should improve rapidly, and the use of $\beta$ as a meeting ground between studies of the CMB and large-scale structure will undoubtedly lead to more demanding tests of the theory in years to come. For the present, we can say that there is complete consistency between clustering in the 2dFGRS and the emerging 'standard model' of cosmology: a spatially flat, vacuum-dominated universe with density parameter $\Omega \simeq 0.3$.

Received 20 December 2000; accepted 30 January 2001.

1. Hubble, E. P. The distribution of extra-galactic nebulae. Astrophys. J. 79, 8-76 (1934).

2. Kirshner, R. P., Oemler, A., Schechter, P. L. \& Shectman, S. A. A million cubic megaparsec void in Bootes. Astrophys. J. 248, L57-L60 (1981).

3. Davis, M. \& Peebles, P. J. E. A survey of galaxy redshifts. V-The two-point position and velocity correlations. Astrophys. J. 267, 465-482 (1983).

4. Bean, A. J., Ellis, R. S., Shanks, T., Efstathiou, G. \& Peterson, B. A. A complete galaxy redshift sample. I-The peculiar velocities between galaxy pairs and the mean mass density of the Universe. Mon. Not. R. Astron. Soc. 205, 605-624 (1983).

5. de Lapparent, V., Geller, M. J. \& Huchra, J. P. A slice of the universe. Astrophys. J. 302, L1-L5 (1986).

6. Kaiser, N. A sparse-sampling strategy for the estimation of large-scale clustering from redshift surveys. Mon. Not. R Astron. Soc. 219, 785-790 (1986)

7. Saunders, W. et al. The density field of the local universe. Nature 349, 32-38 (1991).

8. Shectman, S. A. et al. The Las Campanas redshift survey. Astrophys. J. 470, 172-188 (1996).

9. Taylor, K., Cannon, R. D. \& Watson, F. G. Anglo-Australian Telescope's 2dF facility. Proc. SPIE 2871, 145-149 (1999).

10. Colless, M. First results from the $2 \mathrm{dF}$ galaxy redshift survey. Phil. Trans. R. Soc. Lond. A 357, 105-116 (1999).

11. Maddox, S., Efstathiou, G. \& Sutherland, W. J. The APM Galaxy Survey-III. An analysis of systematic errors in the angular correlation function and cosmological implications. Mon. Not. $R$. Astron. Soc. 283, 1227-1263 (1996)

12. Kaiser, N. Clustering in real space and in redshift space. Mon. Not. R. Astron. Soc. 227, 1-21 (1987)

13. Hamilton, A. J. S., Tegmark, M. \& Padmanabhan, N. Mon. Not. R. Astron. Soc. 317, L23-L27 (2000).

14. Pen, U.-L. Reconstructing nonlinear stochastic bias from velocity space distortions. Astrophys. J. 504, 601-606 (1998)

15. Dekel, A. \& Lahav, O. Stochastic nonlinear galaxy biasing. Astrophys. J. 520, 24-34 (1999).

16. Benson, A. J., Cole, S., Frenk, C. S., Baugh, C. M. \& Lacey, C. G. The nature of galaxy bias and clustering. Mon. Not. R. Astron. Soc. 311, 793-808 (2000).

17. Kauffmann, G., Nusser, A. \& Steinmetz, M. Galaxy formation and large-scale bias. Mon. Not. $R$. Astron. Soc. 286, 795-811 (1997).
18. Strauss, M. A. \& Willick, J. A. The density and peculiar velocity fields of nearby galaxies. Phys. Rep. 261, 271-431 (1995)

19. Hamilton, A. J. S. in The Evolving Universe Vol. 185, 231 (Kluwer Astrophysics and Space Science Library, Kluwer, Dordrecht, 1998); also preprint astro-ph/9708102 at 〈xxx.lanl.gov〉 (1997).

20. Taylor, A. N., Ballinger, W. E., Heavens, A. F. \& Tadros, H. Application of data compression methods to the redshift-space distortions of the PSCz galaxy catalogue. Mon. Not. R. Astron. Soc. (in the press); also preprint astro-ph/0007048 at $\langle x x x . l a n l . g o v\rangle(2000)$.

21. Outram, P. J., Hoyle, F. \& Shanks, T. The Durham/UKST galaxy redshift survey-VII. Redshift-space distortions in the power spectrum. Mon. Not. R. Astron. Soc. (in the press); also preprint astro-ph/ 0009387 at xxx.lanl.gov〉 (2000)

22. Hamilton, A. J. S. Toward better ways to measure the galaxy correlation function. Astrophys. J. 417, 19-35 (1993).

23. Landy, S. D. \& Szalay, A. S. Bias and variance of angular correlation functions. Astrophys. J. 412, 64-71 (1993).

24. Cole, S., Hatton, S., Weinberg, D. H. \& Frenk, C. S. Mock 2dF and SDSS galaxy redshift surveys. Mon. Not. R. Astron. Soc. 300, 945-966 (1998).

25. Feldman, H. A., Kaiser, N. \& Peacock, J. A. Power-spectrum analysis of three-dimensional redshift surveys. Astrophys. J. 426, 23-37 (1994)

26. Meiksin, A. A. \& White, M. The growth of correlations in the matter power spectrum. Mon. Not. R. Astron. Soc. 308, 1179-1184 (1999).

27. Scoccimarro, R., Zaldarriaga, M. \& Hui, L. Power spectrum correlations induced by nonlinear clustering. Astrophys. J. 527, 1-15 (1999).

28. Szalay, A. S., Matsubara, T. \& Landy, S. D. Redshift-space distortions of the correlation function in wide-angle galaxy surveys. Astrophys. J. 498, L1-L4 (1998).

29. Folkes, S. J. et al. The $2 \mathrm{dF}$ galaxy redshift survey: spectral types and luminosity functions. Mon. Not. R. Astron. Soc. 308, 459-472 (1999).

30. Benoist, C., Maurogordato, S., da Costa, L. N., Cappi, A. \& Schaeffer, R. Biasing in the galaxy distribution. Astrophys. J. 472, 452-459 (1996).

31. Loveday, J., Maddox, S. J., Efstathiou, G. \& Peterson, B. A. The Stromlo-APM redshift survey. 2: Variation of galaxy clustering with morphology and luminosity. Astrophys. J. 442, 457-468 (1995).

32. Carlberg, R. G. et al. Galaxy clustering evolution in the $\mathrm{CNOC} 2$ high-luminosity sample. Astrophys. J. 542, 57-67 (2000)

33. Verde, L., Heavens, A. F., Matarrese, S. \& Moscardini, L. Large-scale bias in the Universe-II. Redshiftspace bispectrum. Mon. Not. R. Astron. Soc. 300, 747-756 (1998)

34. Jaffe, A. et al. Cosmology from Maxima-1, Boomerang and COBE/DMR CMB Observations; preprint astro-ph/0007333 at 〈xxx.lanl.gov〉 (2000).

35. Mould, J. R. et al. The Hubble Space Telescope key project on the extragalactic distance scale. XXVIII. Combining the constraints on the Hubble constant. Astrophys. J. 529, 786-794 (2000).

36. Freedman, W. L. et al. Final results from the Hubble Space Telescope key project to measure the Hubble constant; preprint astro-ph/0012376 at 〈xxx.lanl.gov〉 (2000).

37. Ballinger, W. E., Peacock, J. A. \& Heavens, A. F. Measuring the cosmological constant with redshift surveys. Mon. Not. R. Astron. Soc. 282, 877-888 (1996).

38. Hatton, S. \& Cole, S. Modelling the redshift-space distortion of galaxy clustering. Mon. Not. R. Astron Soc. 296, 10-20 (1998).

39. Baugh, C. M. \& Efstathiou, G. The three-dimensional power spectrum measured from the APM galaxy survey-2. Use of the two-dimensional power spectrum. Mon. Not. R. Astron. Soc. 267, 323 332 (1994).

40. Hamilton, A. J. S. Measuring omega and the real correlation function from the redshift correlation function. Astrophys. J. 385, L5-L8 (1992).

41. Jing, Y. P., Mo, H. J. \& Börner, G. Spatial correlation function and pairwise velocity dispersion of galaxies: Cold dark matter models versus the Las Campanas survey. Astrophys. J. 494, 1-12 (1998)

42. Jenkins, A. R. et al. Evolution of structure in cold dark matter universes. Astrophys. J. 499, 20-40 (1998)

43. Peacock, J. A. \& Dodds, S. J. Non-linear evolution of cosmological power spectra. Mon. Not. R. Astron Soc. 280, L19-L26 (1996).

\section{Acknowledgements}

The 2dF Galaxy Redshift Survey was made possible through the dedicated efforts of the staff of the Anglo-Australian Observatory, both in creating the two-degree-field instrument and in supporting it on the telescope.

Correspondence and requests for materials should be addressed to J.A.P. (e-mail: jap@roe.ac.uk). 